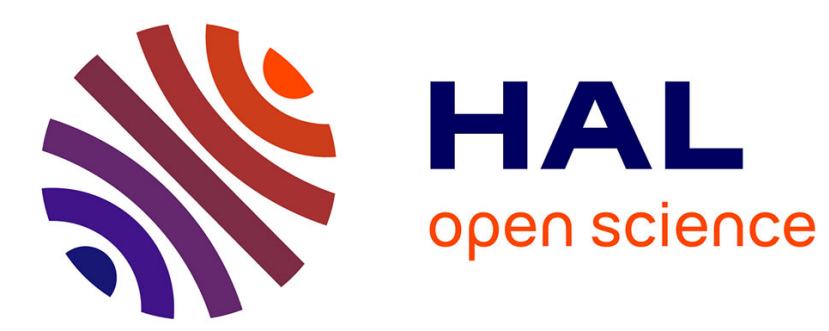

\title{
Le carnet de recherche. Un nouvel outil dans l'écriture d'une histoire du temps présent
}

Malika Rahal

\section{To cite this version:}

Malika Rahal. Le carnet de recherche. Un nouvel outil dans l'écriture d'une histoire du temps présent. Le Mouvement social, 2019, n $^{\circ} 269-270$ (4), pp.133. 10.3917/lms.269.0133 . hal-03095583

\section{HAL Id: hal-03095583 https://hal.science/hal-03095583}

Submitted on 4 Jan 2021

HAL is a multi-disciplinary open access archive for the deposit and dissemination of scientific research documents, whether they are published or not. The documents may come from teaching and research institutions in France or abroad, or from public or private research centers.
L'archive ouverte pluridisciplinaire HAL, est destinée au dépôt et à la diffusion de documents scientifiques de niveau recherche, publiés ou non, émanant des établissements d'enseignement et de recherche français ou étrangers, des laboratoires publics ou privés. 


\title{
Le carnet de recherche. \\ Un nouvel outil dans l'écriture d'une histoire du temps présent
}

par Malika Rahal*

\author{
Le présent texte est le fichier auteur de l'article suivant : \\ M. RAHAL, « Le carnet de recherche. Un nouvel outil dans l'écriture d'une histoire du temps \\ présent », Le Mouvement social, 2019, p. 133-148. \\ Pour toute citation, prière de se référer à la version publiée.
}

\section{Résumé}

Le présent article s'intéresse à la place de la rédaction d'un blog (ou carnet de recherche) dans le travail des historiens, en partant du carnet Textures du Temps, consacré à l'histoire de l'Algérie contemporaine et présent sur la plateforme Hypotheses.org depuis 2012. L'article caractérise l'écriture historienne permise par le blog et la compare aux écritures plus traditionnelles. Il décrit les circulations de textes entre différents supports (du blogs aux revues ou aux médias) ainsi que les liens, en amont et en aval de la mise en ligne, entre réseaux sociaux et blog. Cette écriture renouvelle les relations entre historiens et témoins et ouvre une diffusion de la recherche sous les formes et vers des publics différents, contribuant à une histoire du temps dont l'ambition est d'être désormais mondiale.

\section{Summary}

This article analyzes the experience of research-blogging by focusing on Textures of Time, a history blog hosted on the Hypotheses.org platform since 2012 and dealing with present time Algeria. The article aims to characterize the style of writing developed on the blog to compare it with more traditional forms of history-writing. It describes circulation of texts from one media to the other, from blog to academic journal or to the press, as well as circulations of texts between blog and social media (before or after online publication of blog posts). While blogging transforms relations between historians and witnesses, it also allows for broader circulations of scientific research reaching a different readership, thus supporting the ambition of a present time history that aims of becoming global.

\footnotetext{
* Chargée de recherche à l'Institut d'histoire du temps présent (CNRS, Université Paris 8)
} 
En 2012, à la création du carnet de recherche Textures du temps sur la plateforme Hypotheses.org, l'à-propos du site s'ouvrait en faisant référence au contexte des pays d'Afrique du Nord et, plus largement, du monde arabe, suite aux « printemps arabes ». Il soulignait les possibilités d'ouverture aussi bien que de destruction d'archives auxquelles les événements de 2011 avaient donné lieu1. Le titre du carnet, Textures du Temps - حبكات الزمن, voulait rendre compte des différences dans la densité du temps, des récits et des sources entre périodes de pré- et post-indépendance, mais aussi du sentiment du temps haché, le temps de l'urgence qui semblait encore être celui de 2011-2012 en Algérie, où une ébauche de «printemps » avait eu lieu, dans le Maghreb et, pour tout dire, dans l'ensemble du monde arabe.

Textures du Temps devait accompagner les recherches d'une historienne fraichement recrutée en France au Centre national de la recherche scientifique (CNRS) et affiliée à l'Institut d'histoire du temps présent (IHTP). Il prenait la forme d'un blog : le mot, qui provient de weblog (un journal sur le web), désigne cette forme d'écriture en ligne développée à partir de la fin des années 1990 comme un journal. Un blog se présente sous la forme d'une succession d'entrées (ou billets) rangées de la plus récente à la plus ancienne. Il présente une certaine régularité de publication, sans que celle-ci soit définie, pas plus que ne l'est la longueur des entrées. Il existe des types variés de blog, du simple journal personnel au journal thématique (blogs de voyage, de sport, de photo, blogs liés à une expérience comme la maladie ou le deuil). On a aussi vu, dans les dernières années, se multiplier les blogs de recherche : blogs de chercheurs, de doctorants ou blogs liés à des projets. Les chercheurs se sont progressivement approprié ces outils, notamment en sciences sociales, à partir des années 2000.

Alors que se créait Textures, un blog dédié à l'histoire de la Tunisie ouvrait en 2011 sur la même plateforme, Histoire et Culture dans la Tunisie contemporaine (HCTC), ${ }^{2}$ le carnet de l'historienne Kmar Bendana. Précédemment, je n'avais identifié qu'un seul universitaire bloguant sur l'histoire du Maghreb, Christopher Silver, qui intervient régulièrement depuis 2008 sur la musique juive du Maghreb - d'abord sur Jewish Maghrib Jukeboxe, puis sur Gharamophone. ${ }^{3}$ D'autres blogs ont été créés depuis, liés à des projets particuliers : en 2015, Trajectoires dissidentes, de Pierre-Jean Le Foll-Luciani sur les juifs algériens engagés dans la lutte anticoloniale; en 2019, Native Representations of the French Settlers of Algeria, lié à un projet bénéficiant d'un financement du Arts \& Humanities Research Councilau Royaume-Uni. ${ }^{4}$

Ces exemples rappellent la relative nouveauté et l'hétérogénéité de la pratique du blog pour les chercheurs en sciences sociales, en particulier pour les historiens. Après la période aventurière d'historiens-blogueurs créant et entretenant eux-mêmes leur plateforme et écrivant sous pseudo, ${ }^{5}$ on assiste aujourd'hui à une forme

\footnotetext{
${ }^{1}$ M. RAHAL, «À propos/حبكات الزمن - حن s.d., > >https://texturesdutemps.hypotheses.org/apropos $>$. [Consulté le 25 novembre 2019].

$2<$ https://hctc.hypotheses.org/> [Consulté le 22 novembre 2019].

$3<$ http://jewishmorocco.blogspot.com $>$ et $<$ https://gharamophone.com $>$ [Consultés le 17 octobre 2019].

$4<$ https://www.pieds-noirs.stir.ac.uk/> [Consulté le 22 novembre 2019].

5 Voir l'évocation de cette période par Benoît Kermoal : B. KERMOAL, « Enklask / Enquête, guerre, violences et socialisme (Bretagne, 1900-1940)», Devenir historien-ne, 3 janvier 2013, [En ligne].

<https://devhist.hypotheses.org/1992> [Consulté le 2 décembre 2019].
} 
d'institutionnalisation de la pratique. Depuis 2009, la plateforme Hypotheses.org offre aux universitaires en sciences humaines un hébergement gratuit de ce qui est désormais nommé des « carnets de recherche » plutôt que des « blogs » ${ }^{6}$, sans qu'existe pourtant de différence formelle entre les deux. J'emploie donc les deux termes, « blog de recherche » ou « carnet de recherche », de façon équivalente dans cet article. La plateforme, placée sous la tutelle institutionnelle du CNRS, du ministère français de l'Enseignement supérieur et de la Recherche et de d'Aix-Marseille Université, est dotée d'un conseil scientifique validant la création de chaque carnet. Elle assure aussi une certaine pérennité des contenus, promeut leur caractère « citable » et valorise les textes jugés de qualité. Se distinguent aujourd'hui des carnets d'informations scientifiques (informant sur les colloques, les rencontres, les séminaires etc.), des blogs collectifs destinés à valoriser des projets ou des équipes (blogs de département, de séminaires), ou des blogs individuels permettant de publier des contenus inédits pour présenter et discuter des recherches en cours? . Textures du temps fait partie de cette dernière catégorie, à laquelle s'intéresse le présent article.

Textures du Temps compte, en novembre 2019, 65 billets, rédigés en français, en arabe ou en anglais. Le rythme de publication d'environ neuf billets par an est donc plutôt lent. Ces billets prennent la forme de courts articles, dont plusieurs sont devenus des articles de presse ou de revues scientifiques, montrant le double lien de cette écriture historienne en ligne avec la vulgarisation d'une part et l'écriture scientifique d'autre part. Le carnet reçoit au minimum 130 visites par jour, parfois jusqu'à 400, voire 700 après la publication d'un nouveau billet, selon les données mesurées par Hypotheses.org.

En répondant à la sollicitation des coordinateurs de ce numéro du Mouvement Social de revenir sur l'expérience de ce carnet de recherche, cet article vise à réfléchir à ce qui caractérise l'écriture du blog scientifique en sciences humaines et à décrire comment elle dialogue avec, ou concurrence, d'autres pratiques rédactionnelles, afin de réfléchir à la façon dont l'animation d'un carnet peut s'intégrer dans la vie de la recherche. Nous reviendrons d'abord sur ce que permet l'écriture de blog dans le domaine particulier de l'historiographie de l'Algérie coloniale et en quoi elle est - ou non - une solution à certaines difficultés de l'histoire du temps présent du Maghreb contemporain. Nous apporterons ensuite des éléments de comparaison avec d'autres écritures historiennes. Enfin, nous examinerons la diffusion des textes et leurs circulations entre différents supports (réseaux sociaux, revues scientifiques, médias).

\section{Développer l'historiographie de l'Algérie contemporaine}

À ses débuts en 2012, Textures du Temps devait accompagner une recherche individuelle

\footnotetext{
${ }^{6}$ F. HEIMBURGER et É. RuIZ, « Has the Historian's craft gone digital? Some observations from France », Diacronie. Studi Di Storia Contemporanea, 2012.; É. RuIz, « L'écriture de l'histoire a-t-elle connu un tournant numérique? (\#METSEM 23) », La boîte à outils des historien-ne:s, $1^{\mathrm{er}}$ juillet 2019, [En ligne].

<https://www.boiteaoutils.info/2019/07/metsem23/> [Consulté le 26 novembre 2019].

7 Je n'en cite que deux exemples : Acquis de Conscience $<$ https://consciences.hypotheses.org/>, le blog de l'historienne Caroline Muller depuis son doctorat, et Montaincoastriver

$<$ http://mountaincoastriver.blogspot.com/>, le blog de l'historien David Gange, autour d'un projet unique [Consultés le 22 novembre 2019].
} 
à un moment particulier : après avoir travaillé sur l'Algérie coloniale, j'entrais au CNRS avec un programme de recherche incluant l'Algérie post-indépendance - une histoire marquée par un fort déficit d'historiographie, un accès difficile, sinon impossible, aux archives nationales, et les réticences des témoins dans un contexte où les enjeux politiques autour de l'usage de l'histoire demeurent forts. ${ }^{8} \mathrm{C}$ 'était un champ de recherche solitaire et le blog avait un double but: accompagner la réflexion sur les façons de surmonter ces difficultés et promouvoir un champ auprès de futurs chercheurs en partageant idées ou trouvailles archivistiques.

\section{Contourner le déficit historiographique}

Une grande partie de mon temps de recherche était alors consacrée à la rencontre de témoins et à l'exploration de toutes les alternatives aux archives nationales'. Beaucoup de sujets de recherches peuvent échouer fautes d'archives ou d'accès aux témoins. J'ai donc choisi d'explorer parallèlement plusieurs sujets avant de déterminer lesquels seraient réalisables, tout en laissant sur le blog des traces de ces explorations comme autant de jalons de la réflexion, susceptibles de stimuler de futurs chercheurs. Ces observations et découvertes étaient encore insuffisamment travaillées pour être présentées en conférence ou dans les sections des revues d'histoire consacrées aux archives, mais pouvaient inspirer des collègues, dans une «logique de partage $\gg^{10}$. Textures du temps a ainsi présenté différents fonds d'archives, du fonds Cohen-Addad déposé à l'IHTP et qui comprend des photographies de l'indépendance, ${ }^{11}$ aux archives nationales américaines où l'on trouve les rapports des consuls américains en Algérie et dont, avec Arthur Asseraf, nous avons essayé de donner les clefs de l'organisation kafkaïenne, en passant par les archives du Comité international de la Croix-Rouge et du Croissant-Rouge (CICR) à Genève. ${ }^{12}$

On touche ici à l'une des caractéristiques du carnet de recherche, souvent mise en avant par les blogueurs : le partage de textes à mi-chemin entre les notes ou le journal de recherche que l'on rédige pour soi et la publication scientifique. Rédiger un billet peut donc constituer une première étape de stabilisation de réflexions précoces ou de présentation de premiers résultats, et le blog fonctionne comme un incubateur ${ }^{13}$. Plusieurs billets ont d'ailleurs ensuite fait l'objet d'invitations à présenter le travail dans des conférences ou séminaires, et ces discussions ont parfois permis de reprendre des pistes un temps abandonnées. De ce point de vue, l'animation d'un blog s'adaptait bien à

\footnotetext{
${ }^{8}$ M. RAHAL, « Fused Together and Torn Apart: Stories and Violence in Contemporary Algeria », History \& Memory, 2012, p. 118-151.

9 M. RAHAL, « Comment faire l'histoire de l'Algérie indépendante? », La Vie des Idées, mars 2012.

${ }^{10}$ E. POUPARDin et M. FAURY, « Hypotheses : l'inscription d'une pratique de communication dans l'activité de recherche », Revue française des sciences de l'information et de la communication, décembre 2018.

${ }^{11}$ M. RAHAL, « Bons baisers d'Alger, 1962 », Textures du temps - حبكات الزمن 2019 mai 2013,

$<$ https://texturesdutemps.hypotheses.org/757> [Consulté le 4 juillet 2019].

${ }^{12}$ A. ASSERAF et M. RAHAL, « Writing a history of independent Algeria in America? The myth of the archive », Textures du temps- حبكات الزمن, 20 janvier 2016, <https://texturesdutemps.hypotheses.org/1851> [Consulté le 30 mars 2019].

${ }^{13}$ P. Mounier, « Ouvrir l'atelier de l'historien : médias sociaux et carnets de recherche en ligne », Homo Numericus, 9 novembre 2011, <http://www.homo-numericus.net/article304.html> ; A. GUNTHERT, « Why blog? », Actualité de la Recherche en histoire visuelle, 15 septembre 2008, [En ligne]

<http://www.arhv.lhivic.org/index.php/2008/09/15/807-why-blog> [Consultés le 27 novembre 2019].
} 
la phase exploratoire de l'entrée dans le métier de chercheuse, tout en lui donnant une dimension publique.

\section{Constituer une communauté de chercheur}

Dans un contexte où les recherches historiques sur le Maghreb contemporain sont devenues très faibles en France et encore insuffisantes dans les pays du Maghrebmême ${ }^{14}$, l'idée s'est imposée rapidement d'utiliser Textures du Tempspour donner à voir une communauté de chercheurs en formation. Masterants, doctorants voire chercheurs plus avancés ont été invités à s'aventurer au-delà du seuil de l'indépendance en développant des textes parfois à la marge de leurs recherches, et en tout cas non encore publiées. À ce stade, onze auteurs différents ont signé des billets sur le carnet de recherche, dont un collectif de chercheurs.

En chemin, il est apparu que l'attractivité du blog variait d'une personne à l'autre et d'une culture académique à l'autre. Ceux qui ont accepté le plus volontiers l'invitation ont été des chercheurs dont le travail est encore peu connu et la thèse non encore achevée. Outre le plaisir de cette forme d'écriture, ils y ont trouvé un autre moyen de faire connaître leurs travaux dès avant leur soutenance et la candidature à des postes universitaires. Les contributeurs ont souvent été séduits par la rapidité et la légèreté de l'écriture et de la publication par rapport aux revues papier ou en ligne. Le caractère «citable » des billets est également rassurant et certains de ces textes d'invités sont parmi les plus cités du blog, à l'instar de la série proposée par Ed McAllister sous le titre « Algeria's Belle Epoque » sur la nostalgie des années Boumediene : la thèse de l'auteur n'est toujours pas publiée mais la série est régulièrement mentionnée, y compris dans des articles de revue ${ }^{15}$.

L'absence de processus de peer-review peut expliquer pourquoi d'autres auteurs n'acceptent pas l'invitation, même si leurs motivations restent généralement tues. Peutêtre cette publication est-elle également jugée moins attractive? Seule une doctorante qui, en Algérie, préparait une belle monographie de village de colonisation s'est inquiétée du fait que son directeur de thèse pourrait désapprouver la publication sur un blog. Le fait est qu'aucun chercheur ou doctorant algérien n'a accepté cette invitation à écrire, déjouant ainsi l'ambition originelle de faire connaître des travaux en cours en Algériemême, en particulier en langue arabe. Les collègues britanniques et américains ont été les plus enthousiastes (cinq contributeurs) alors que les collègues travaillant en France sont souvent réservés (trois contributeurs seulement). Il est vraisemblable que ce qui

\footnotetext{
${ }_{14}$ N. Amara, C. RAYMond, et J. SFEIR, « Présentation de Écritures historiennes du Maghreb et du Machrek », Naqd, 2014 ; M. Oualdi, I. Grangaud, et A. MesSAOUdi, « Dossier de recherche X | 2014 : Besoins d'Histoire. Historiographies et régimes d'historicité au Maghreb à l'aune des révolutions arabes », L'Année du Maghreb, avril 2013 ; F. BEN SLIMANE et H. ABDESSAMAD, La périodisation dans l'écriture de l'histoire du Maghreb. Actes, table ronde de Marrakech les 26-29 mai 2005, table ronde de Tunis les 21-23 septembre 2005, Tunis, Arabesques éditions, 2010.

${ }^{15}$ E. MCAllister, « Algeria's Belle Époque (1) Postcards and Nostalgia: images of Algiers in the 1970s », Textures du temps - لزمن 12 mai 2012, > https://texturesdutemps.hypotheses.org/414> ; « Algeria's Belle Époque (2) Groundhog Day », Textures du temps- حبكات الزمن, 22 avril 2013, [En ligne]

$<$ https://texturesdutemps.hypotheses.org/704> ; « Algeria's Belle Époque (3) Narratives of Religious and Social Change », Textures du temps - حبكات الزمن 30 juin 2013, <https://texturesdutemps.hypotheses.org/836> [Consultés le 18 décembre 2018].
} 
peut apparaître attractif aux uns (le style plus direct, l'écriture à la première personne et la préoccupation de vulgarisation) peut caractériser pour d'autres une écriture moins scientifique, voire marquée par une impudique autopromotion. En France, le temps n'est pas si lointain où l'écriture de blog avait franchement mauvaise presse ${ }^{16}$, et les résistances à la valorisation du blogging scientifique demeurent, notamment à l'étape de l'habilitation à diriger des recherches.

$\mathrm{Au}$ final, l'objectif de faire connaître une communauté de chercheurs travaillant sur l'Algérie contemporaine ne fonctionne que dans la mesure où les collègues souhaitent s'en saisir et demande donc à être davantage travaillé, voire à évoluer vers des formes plus classiques de numéros spéciaux de revue ou de conférences pour constituer un meilleur reflet du champ.

\section{Valoriser la place des témoins}

Enfin, dans le contexte d'une histoire du temps présent où les témoins, leurs témoignages et leurs archives jouent un rôle essentiel, le blog a des effets sur la recherche elle-même. Les billets précocement mis en ligne peuvent en effet modifier les relations entre historien et témoins. Plusieurs billets ont ainsi suscité des échanges avec des témoins déjà interviewés (prolongeant ainsi l'entretien) ou encore inconnus (ouvrant à de nouveaux entretiens). La mise en ligne a parfois permis d'obtenir des réactions sur les billets, voire d'élucider certaines questions.

De tels échanges ne sont pas sans poser de questions méthodologiques : sait-on qui se cache dernière certains pseudonymes? Ne risque-t-on pas de s'enfermer dans des discussions d'entre-soi avec des personnes qui suivent notre travail? Le partage de l'internet algérien entre arabophones et francophones pèse lourdement, mais ce n'est pas le seul clivage. Néanmoins, ces échanges donnent régulièrement lieu à des rencontres et des entretiens formels. Par ailleurs, on objectera que les biais existent également lorsqu'on recherche des témoins par d'autres méthodes, notamment les rencontres par effet boule-de-neige (chaque entretien conduisant à un ou plusieurs autres) ou par le biais de contacts qui nous ouvrent des portes. Les mêmes outils critiques s'appliquent donc à nos méthodologies, anciennes et nouvelles.

Il est plus significatif que l'écriture en ligne permette la création d'un espace modifiant le rapport entre historien et témoin. Elle permet une forme d' empowerment, ouvrant aux témoins connectés l'accès à une recherche jusque-là plutôt cantonnée à des revues scientifiques difficilement accessibles. Surtout, elle leur ouvre la possibilité de répondre, au moins dans la section « commentaire » de chaque billet, sur les réseaux sociaux ou leurs propres blogs. Ces relations directes entre historiens et témoins n'ont pas été inventées par l'écriture en ligne : en France, l'IHTP a une longue histoire de tels échanges concernant Seconde Guerre mondiale et la Résistance. Limitées par la distance, ces expériences ne pouvaient cependant pas être reproduites par des historiens travaillant

\footnotetext{
${ }^{16}$ Voir la description que fait Paul Bertrand de son activité de blogging historien au début des années 2000 : P. BERTRAND, « Pourquoi Médiévizmes? », Devenir historien-ne, 21 décembre 2012, [En ligne]

<https://devhist.hypotheses.org/1981>. [Consulté le 2 décembre 2019].
} 
sur d'autres pays, alors même que la restitution de la recherche aux témoins qui l'ont rendu possible est fondamentale pour celles et ceux qui pratiquent une histoire à base d'entretiens et de collectes de sources privées, plus encore peut-être dans les cas d'historiens travaillent dans un pays du Nord pour étudier un pays du Sud ${ }^{17}$. Ici, la publication en ligne élargit des contacts et peut participer à « égaliser » l'échange entre historien et témoin, à contrebalancer les relations de pouvoir qui existent entre eux.

Il ne s'agit donc aucunement de sombrer dans le fétichisme d'un instrument technique qui viendrait nous sauver de maux anciens, mais de voir comment l'écriture de blog en amont de la publication classique peut favoriser une histoire du temps présent renouvelée, avec un plus grand potentiel pour devenir véritablement globale.

\section{Un autre type d'écriture historienne?}

$\mathrm{Au}$-delà de cette contribution à la résolution des difficultés historiographiques, le blog historien s'avère aussi être le support d'expérimentation de styles d'écriture différents, d'une « écriture libérée ${ }^{18}$, rendue visible par la variété des styles de billets de Textures du Temps. Tentons donc d'extraire certaines caractéristiques communes aux textes qui attirent le plus de lecteurs et suscitent le plus de commentaires.

\section{Construire une mise en récit}

L'écriture sous forme de billets de blog, conçue dans Textures sous la forme de textes parfois longs mais destinés à un large public, a des caractéristiques qui diffèrent de l'écriture scientifique plus classique. Les billets courts comptent 7 à 9000 signes; plus fréquemment, ils comprennent entre 15000 et 20000 signes. L'une de leur caractéristique réside dans l'importance de la mise en récit.

Raconter une histoire, privilégier un personnage, pointer une anecdote permet à des billets, mêmes longs, d'être lus. Les questions théoriques n'y sont pas abordées frontalement mais émanent de l'histoire racontée, sans longs développements et sans jargon. Entre les deux sens du mot français histoire (history-the story; الحكاية-التاريخ ; Geschichte-Historie), les billets font donc volontiers pencher la balance vers le « récit de ces séries d'événements attribués à des noms propres », selon l'expression de Jacques Rancière ${ }^{19}$, plutôt que vers une histoire préoccupée d'énoncer sa propre scientificité.

Cette centralité du récit modifie l'organisation des textes. En France, la structure hiérarchisée en parties et sous-parties (généralement par trois) demeure un idéal de l'écriture historienne et peut se faire au détriment des histoires mobilisées pour appuyer l'argumentation, qui risquent de la déséquilibrer. $A$ contrario, les billets de blog s'organisent de façon plus linéaire que pyramidale, le rythme y est central et, bien

17 Dans un contexte différent de celui du Maghreb, voir L.T. SMith, Decolonizing Methodologies: Research and Indigenous Peoples, 2d edition., London, Zed Books, 2012. Voire aussi l'étude consacrée aux enquêtés du camp de réfugiés palestiniens au Liban de Shatila : M. SUKARIEH et S. TANNOCK, « On the Problem of Over-researched Communities: The Case of the Shatila Palestinian Refugee Camp in Lebanon », Sociology, 2013, p. 494-508. ${ }^{18}$ O. DeseillignY, «Matérialités de l'écriture : le chercheur et ses outils, du papier à l'écran », Sciences de la société, octobre 2013, p. 38-53.

19 J. RANCIÈRE, Les noms de l'histoire. Essai de poétique du savoir, Paris, Seuil, 1992. 
souvent, c'est le récit qui impose la structure dans laquelle viendront se loger concepts et arguments théoriques, plutôt que l'inverse.

Par ailleurs, ces billets intègrent volontiers, outre les références historiennes, des références ou citations de romans, poèmes, extraits de films ou photographies qui permettent de visualiser les lieux d'aujourd'hui. Certains carnets de recherche encouragent les contributeurs à remplacer les notes par des liens, pour une lecture plus fluide. ${ }^{20}$ Je privilégie pour ma part un référencement précis en notes, qui n'empêche pas les liens hypertextes. Ces citations sont cependant traitées autrement qu'elles ne le seraient dans une revue scientifique et c'est, à mon sens, l'un des éléments de cette « écriture libérée » propre au blog. Ainsi, la référence à deux romans, Les Chercheurs d'Os de Tahar Djaout et Le Général de l'Armée morted'Ismaill Kadaré, est utilisée dans un billet sur les disparus de la Guerre d'Indépendance pour signaler la différence des conditions de la recherche des corps avec la Première Guerre mondiale. ${ }^{21}$ Dans un article de revue, le statut de ces références littéraires devrait être élucidé et s'accompagner de références à l'abondante littérature historienne sur le sujet pour acquérir valeur de preuve. Dans le billet de blog, elle tient du raccourci et de l'ouverture à d'autres lectures. Leur évocation prend volontiers un tour poétique, contribuant à donner au texte une tonalité littéraire que nos articles de science trouvent moins fréquemment.

\section{Le récit de soi}

Parmi les billets les plus lus du carnet se trouvent ceux dans lesquels l'auteur se met en scène à une étape donnée de sa recherche, dans son rapport au terrain et ses interrogations méthodologiques. Cette ouverture de l'atelier de l'historien permet aux lecteurs de suivre le cheminement de l'auteur ${ }^{22}$. Pour autant, elle n'est pas limitée à l'amont de la recherche mais peut aussi se positionner en aval, pour historiciser les circonstances même de la recherche: ainsi, un billet concernant la rédaction de la biographie d'Ali Boumendjel, militant assassiné durant la « Bataille d'Alger », raconte une expérience d'histoire du temps présent, interrogeant les rapports avec les témoins, la justice et la vérité dans le contexte de la France des années $2000^{23}$.

Le risque de cette approche est que cette « éditorialisation de soi $»^{24}$ ne prenne le pas sur la nécessaire distance critique. Pour autant, cette dimension ego-historienne correspond aussi à une étape indispensable de la réflexion, qui devrait intervenir tôt dans toute recherche, en particulier lorsqu'elle concerne des événements suffisamment récents

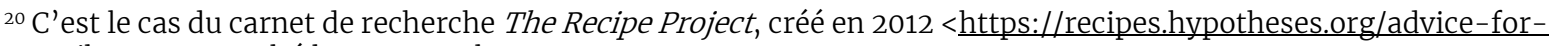
contributors, consulté le 20 novembre 2019>.

${ }^{21}$ M. RAHAL, «A la recherche des disparus de la guerre d'indépendance », Textures du temps- حبكات الزمن août 29 aove 2017, <https://texturesdutemps.hypotheses.org/2506> [Consulté le 21 novembre 2019].

22 Sur cette question de la révélation de l'atelier de l'historien.ne, voir notamment A. GUNTHERT, « Why blog? », op. cit. ; M. DAcos et P. MOUNIER, « Les carnets de recherche en ligne, espace d'une conversation scientifique décentrée », Lieux de savoir. T.2. Les mains de l'intellect, Christian Jacob., Paris, Albin Michel, 2010, p. ; P. BERTRAND, « Pourquoi Médiévizmes? », op. cit.; B. KeRMOAL, « Enklask / Enquête, guerre, violences et socialisme (Bretagne, 1900-1940) », op. cit.

${ }_{23}$ M. RAHAL, « La terrasse. Retour sur une histoire du temps présent », Textures du temps- حبكات الزمن , 16 août 2015 , $<$ https://texturesdutemps.hypotheses.org/1108>. [Consulté le 21 novembre 2019]; au sujet du livre M. RAHAL, Ali Boumendjel. Une affaire française, une histoire algérienne, Paris, Belles Lettres, 2010.

24 E. POUPARDIn et M. FAURY, « Hypotheses », op. cit.
} 
pour marquer les parcours des chercheurs qui les étudient. En France, malgré un ouvrage canonique et la publication récente de plusieurs mémoires de synthèse d'habilitation à diriger des recherches ${ }^{25}$, l'intégration de la dimension ego-historienne dans les publications de recherche n'est pas une évidence. Le blog fournit l'occasion régulière de ce pas de côté réflexif. C'est par exemple le cas sur le blog de l'historien Benjamin T. White, Singular Things, dont le titre est emprunté à Marc Bloch défendant, dans Le Métier de l'historien, « la volupté d'apprendre des choses singulières » ${ }^{26}$. Les lecteurs y découvrent des aspects méconnus du métier d'historien aux travers de réflexions de voyages (l'un d'eux au camp de Rivesaltes) ${ }^{27}$, sur le rythme de la publication, le processus de peer-reviewing ou les liens entre recherches passées et actualité28. Les auteurs de Textures du Temps écrivent eux aussi à partir de lieux visités, de documents découverts, d'ouvrages lus ou de rencontres avec des témoins. Ils évoquent la place de l'historien dans sa recherche, soulevant certaines grandes questions de l'histoire du temps présent.

Nombre de ces billets de blog, par leur style et leur ton, sont proches de ceux que les anglophones nomment un essay: un texte de non-fiction dans lequel l'auteur.e s'autorise un point de vue personnel. Ils sont ce que Dan Coen nomme des blessays, mêlant blog et essay ${ }^{29}$. La préexistence de l'essay dans le monde anglophone semble faciliter l'acceptation la rédaction d'un blog comme activité légitime dans la recherche. Malgré le point de vue personnel - ou peut-être grâce à lui -, ces billets posent des questions éminemment historiennes, tout en parlant à un lectorat non-universitaire qui, comme le montrent les commentaires des lecteurs de Textures du Temps, apprécie l'humilité des auteurs, comme s'ils sortaient ainsi d'une position de surplomb académique en devenant les personnages accessibles de leurs propres recherches.

\section{Porter sur l'actualité un regard d'historien}

Enfin, par sa forme de journal, le blog invite à une écriture à laquelle les historiens sont plus rétifs que d'autres chercheurs, une écriture réactive à l'actualité.

Sur Textures $d u$ Temps, ce rapport à l'actualité s'est vérifié depuis le début du mouvement populaire, le ḥirāk [الحراك الثعبي] qui a démarré en Algérie en février 2019. Quatre billets ont été publiés par trois auteurs différents entre le 4 et le 19 mars 2019, un rythme inhabituellement rapide. Dans l'un d'eux, l'historienne Natalya Vince anticipait la troisième manifestation prévue le 8 mars, qui allait correspondre à la journée internationale du droit des femmes et laissait présager l'entrée massive des Algériennes dans le mouvement. À partir de recherches passées, elle retraçait une brève histoire du 8

\footnotetext{
${ }_{25}$ Voir P. Nora (dir.), Essais d'ego-histoire, Paris, Gallimard, 1987. La publication de ces mémoires dits « de synthèse » est l'objectif de collection Itinéraires, désormais dirigée par Patrick Boucheron, aux éditions de la Sorbonne.

${ }^{26}$ M. BLOCH, Apologie pour l'histoire ou métier d'historien, Paris, Armand Colin, 2018.

27 B.T. WHITE, « A camp in France », Singular Things, 28 juin 2017, [En ligne].

<https://singularthings.wordpress.com/2017/06/28/a-camp-in-france/> [Consulté le 21 novembre 2019].

${ }_{28}^{8}$ B.T. WHITE, « Revise and resubmit », Singular Things, 16 juin 2019, [En ligne].

$<$ https://singularthings.wordpress.com/2019/06/16/revise-and-resubmit/> [Consulté le 21 novembre 2019].

29 D. CoHEN, « The Blessay », Dan Cohen, 24 mai 2012, [En ligne] <https://dancohen.org/2012/05/24/the-blessay/> [Consulté le 16 octobre 2019].
} 
mars en Algérie $^{30}$. Cette mobilisation de recherches existantes pour éclairer les événements en cours est l'une des façons dont les historiens peuvent aborder l'actualité. Avec leur regard d'historiens, les auteurs interrogent aussi les sources pour connaître le mouvement ${ }^{31}$, ainsi que les notions d'événement, d'héritage ou de durée ${ }^{32}$, produisant des textes qui diffèrent d'autres textes écrits dans les médias à la même époque. Ces réactions à l'actualité peuvent également prendre la forme d'interventions en réaction aux usages médiatiques de l'histoire, toujours fondées sur l'expertise historienne de leurs auteurs, comme c'est le cas dans deux tribunes de Natalya Vince décryptant les sous-entendus historiques de plusieurs grands journaux britanniques après une prise d'otage à Aïn Amenas dans le Sud algérien en janvier 2013 et après les attentats de novembre 2015 en France ${ }^{33}$. Ces textes, refusés par les journaux concernés, prolongent des réflexions académiques menées notamment par l'historien James McDougall pour déconstruire les représentations de l'Algérie comme un pays voué à une violence à perpétuité, et ouvrent ces débats universitaires à un public plus large ${ }^{34}$.

Si certains de ces billets d'actualité ressemblent à des tribunes, leur style reflète aussi les liens entre recherche de longue haleine et immédiateté en distinguant les faits confirmés et les hypothèses. L'écriture admet aussi l'aveu des doutes ou du malaise de l'historien face à l'événement. Par ailleurs, l'actualité à laquelle les auteurs réagissent n'est pas toujours médiatique. En cela, le blog tient le rôle du journal où l'on peut relire la trace des événements et de notre propre cheminement intellectuel.

\section{Des textes qui circulent}

Après sept années d'existence, il est possible de cartographier la circulation des textes ou bribes de textes de Textures du temps, du blog aux publications scientifiques, du blog aux grands médias, mais également entre réseaux sociaux et blog, pour toucher des publics multiples. Ces circulations révèlent comment le blog s'intègre dans une constellation de pratiques d'écritures de plus en plus labiles.

\section{Rendre la recherche accessible}

Les chiffres de consultation de Textures du Temps - fournis par Hypotheses.org et qui doivent être maniés avec précaution du fait de l'existence de robots qui les gonflent artificiellement - indiquent environ 180 visites par jour et plus de 5200 visites par mois.

\footnotetext{
${ }^{30} \mathrm{~N}$. Vince, «Les femmes dans la rue : le 8 mars dans la longue histoire de l'Algérie indépendante », Textures du Temps- حبكات الزمن, 7 mars 2019, <https://texturesdutemps.hypotheses.org/3742> [Consulté le 31 mai 2019].

${ }^{31}$ M. RAHAL, « Le peuple et le sens de l'histoire », Textures du temps- حبكات الزمن النكات 4 mars 2019,

<https://texturesdutemps.hypotheses.org/3704> [Consulté le 4 décembre 2019].

32 A. ASSERAF, « Faire peuple à Alger Textures du temps - حبكات الزمن , 19 mars 2019,

<https://texturesdutemps.hypotheses.org/3782> [Consulté le 4 décembre 2019].

33 N. VINCE, « In Amenas - a history of silence, not a history of violence », Textures du temps- حبكات الزمن 20 janvier 2013, <https://texturesdutemps.hypotheses.org/576>.; et « Dangerous shortcuts: Paris attacks and the War of Independence », Textures du temps- حبكات الزمن, 22 novembre 2015, <https://texturesdutemps.hypotheses.org/1754> [Consultés le 25 novembre 2019].

34 J. McDougALL, « Savage Wars? Codes of Violence in Algeria, 1830s-1990s », Third World Quarterly, 2005, p. 117-131.
} 
Ils révèlent aussi 800 à 2000 visites supplémentaires dans les jours qui suivent la mise en ligne d'un billet, correspondant à des visiteurs venus regarder spécifiquement le dernier billet en date. Ces visites proviennent majoritairement de France, d'Algérie et des ÉtatsUnis. Si nous connaissons mal ce lectorat, certains échanges font découvrir des lecteurs réguliers qui, par ailleurs, ne lisent ni d'articles de revues ni d'ouvrages d'histoire.

Ces chiffres de consultation permettent de valider un choix effectué très tôt : celui de proposer des textes dans plusieurs langues, arabe, français et anglais, afin de toucher un public divers. Ils remettent en question certaines idées reçues sur le lectorat dans le monde arabe, où l'on entend souvent dire que le lectorat arabophone ne lit pas de sciences humaines, confirmant qu'il n'en est rien : quoique peu nombreux, les textes en arabe ne sont pas moins lus que les textes en français. L'une des difficultés majeures du travail sur l'Algérie est celle du multilinguisme: la montée dans le pays de générations très éduquées, majoritairement en arabe, réduit le lectorat francophone, cependant que les difficultés matérielles liées à la traduction des articles ou livres rendent difficile l'accès à cette littérature. Par ailleurs, les chercheurs anglo-saxons prennent une place plus importante dans les études sur le Maghreb, publiant et enseignant en anglais, complexifiant encore la question de la circulation et de la traduction des textes.

Or, le carnet de recherche offre, grâce à sa légèreté, quelques solutions à moindre de frais en utilisant les compétences disponibles. Les textes de blogs sont relativement courts, souvent écrit dans un langage accessible; par ailleurs l'absence de deadline et la possibilité de corriger aisément d'éventuelles erreurs confirment la souplesse d'utilisation. Cette écriture permet d'oser davantage. Pour ma part, j'ai donc traité la réécriture en arabe du billet «La Terrasse » comme un exercice, étalé sur plusieurs mois ${ }^{35}$; d'autres billets ont été traduits par un lecteur volontaire. Certains contributeurs écrivent en anglais, j'ai pu les traduire en français et réécrire certains de mes billets en anglais. Les collègues enseignant au Royaume-Uni ou aux États-Unis les utilisent désormais dans leurs enseignements, car les matériaux en langue anglaise manquent cruellement. Dans le choix de la langue d'écriture aussi, le blog de recherche est un lieu d'expérimentation : toute traduction systématique des billets nécessiterait une force de travail ou des financements qui manquent. L'aléatoire constitue une force.

La traduction n'est cependant que l'un des obstacles à la diffusion du savoir. En Algérie comme dans l'ensemble du monde arabe, la diffusion des publications se heurte à des difficultés matérielles, comme le faible nombre de librairies et la faiblesse de la diffusion. La règlementation sur les livres rend difficile l'importation et l'exportation. Ces difficultés expliquent le spectaculaire succès populaire du Salon international du Livre d'Alger ${ }^{36}$. Pourtant, rendre nos travaux accessibles aux personnes concernées est une exigence éthique et politique fondamentale, qui prend une dimension particulière dans la situation de chercheurs travaillant dans des pays du Nord - et parfois dans les

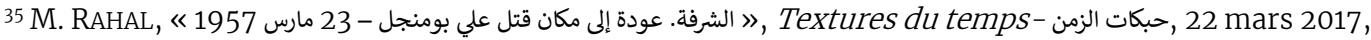
<https://texturesdutemps.hypotheses.org/2418> [Consulté le 22 mars 2017].

36 « Sharjah 2.23 million. Algiers 2.2 million. 4.4 million visitors to Arab book fairs in the past two weeks. The Arab Renaissance gathers momentum », The New Publishing Standard, 12 novembre 2018, [En ligne].

$<$ https://thenewpublishingstandard.com/sharjah-2-23-million-algiers-2-2-million-4-4-million-visitors-arabbook-fairs-past-two-weeks-arab-renaissance-gathers-momentum/> [Consulté le 2 décembre 2019].
} 
anciennes puissances coloniales des pays qu'ils étudient. Face à ces difficultés d'accès au savoir, l'écriture en ligne, en particulier l'écriture de blog, offre des solutions puissantes grâce au développement de la $3 \mathrm{G}$ puis de la $4 \mathrm{G}$ au Maghreb ces dernières années ${ }^{37}$. Les islamistes de tous bords le savent, qui constituent d'ailleurs des bibliothèques gratuites d'ouvrages sur internet. Penser l'accessibilité des textes de sciences sociales est donc fondamental, et le blog n'est que l'une des façons de procéder, révélant un lectorat friand, composé de non-universitaires, d'étudiants et de collègues.

Les statistiques d'Hypotheses.org rendant visibles les modalités de l'accès des lecteurs au carnet, nous savons que jusqu'à $80 \%$ des visiteurs sont parvenus à un billet par le biais de réseaux sociaux, à une écrasante majorité Facebook. Les lecteurs dépendent donc aujourd'hui des réseaux sociaux plutôt que d'autres technologies, comme l'agrégation de flux RSS, pour arriver au blog. Partant de ce constat, il est possible d'avoir une politique de diffusion consistant à partager les billets à leur parution et de prolonger la durée de vie de chaque billet à le faisant circuler de nouveau en réaction à l'actualité ou à une date anniversaire. Cette dépendance, qui conforme le pouvoir des GAFA, les géants du web, dans la circulation du savoir, est néanmoins préoccupante. Elle fait écho à leurs politiques agressives dans les pays du Sud et en particulier sur le marché algérien, où des packs peu couteux sont proposés par les fournisseurs de téléphonie mobiles qui offrent un accès internet modeste mais un accès illimité à Facebook : ils encouragent alors la consultation de contenu par le biais du réseau social. L'une des conséquences de cette dépendance est que les discussions autour des billets ont lieu non directement sur le blog, mais sur les réseaux sociaux. De ce fait, leur consultation et leur archivage ne sont pas toujours assurés ${ }^{38}$.

\section{Du blog aux publications scientifiques}

La question de la diffusion des textes et de leur circulation pose ensuite la question des rapports entre blog et publications scientifiques plus classiques, pour déterminer s'il y a là concurrence ou synergies entre plusieurs types d'écriture.

Le cas d'une série de deux billets interroge la relation entre écriture de blog et d'articles. Rédigés après l'annonce, en janvier 2017, de la fin des travaux de déminage des engins explosifs laissés par l'armée française après la Guerre d'Indépendance, ils rassemblaient à la fois quelques archives classiques sur cette question, additionnées de documents disponibles en ligne et liées à l'adhésion en 2000 de l'Algérie à la Convention d'Ottawa contre les mines antipersonnel ${ }^{39}$. Après la mise en ligne, des lecteurs ont signalé d'autres documents en ligne, ajoutés ultérieurement au billet (ce que ne permettrait ni une

\footnotetext{
37 InternetWorldStat évalue l'accès à internet à 44\% de la population totale en Algérie, 92,6\% en France et 89,7\% aux États-Unis en 2017 <https://www.internetworldstats.com/> [Consulté le 12 décembre 2018].

En octobre 2019, il était possible en Algérie d'acheter avec l'un des principaux fournisseurs de téléphonie mobile un forfait à $200 \mathrm{DA}$ (environ $1,5 €$ ) pour 3 mégaoctets de données et valable une journée.

${ }^{38}$ Pour un exemple de ce type, voir les commentaires d'un billet intégrant des échanges avec les historiens Henry Rousso et Denis Peschanski, extraits de Facebook : B.T. WHITE, « A camp in France », op. cit.

39 M. RAHAL, « La guerre après la guerre. La fin des opérations de déminage lancées en 1963 », Textures du temps20 29 janvier 2017, > https://texturesdutemps.hypotheses.org/2321> ; et « La guerre après la guerre. Déminage (II) », Textures du temps - حبكات الزمن 16 février 2017, <https://texturesdutemps.hypotheses.org/2401> [Consultés le 25 novembre 2019].
} 
publication papier, ni d'autres types publications électroniques). Même avant ces ajouts, la matière rassemblée aurait pu donner lieu à un article académique et pose la question de savoir si le carnet peut « siphonner » la matière de nos recherches, en réduisant notre productivité en articles de revue.

D'autres exemples indiquent plutôt qu'une publication n'empêche pas l'autre. Des billets de blog de Benjamin T. White consacrés aux stations de quarantaine en Australie ont, par exemple, été repris pour un chapitre d'ouvrage très proche des billets d'origine ${ }^{40}$. Ont seulement été ajoutées une introduction et une problématisation, avec des références situant l'article dans un champ de recherche, tandis que le « je » utilisé dans les billets pour évoquer le présent de la visite des lieux a disparu. Dans le cas d'un article de Textures, la transformation d'un billet en article de revue s'est effectuée en conservant l'écriture d'origine, lorsque les coordinateurs d'un numéro spécial de L'Année du Maghreb ont considéré que le thème et le ton décalé fonctionnaient bien dans l'ensemble du numéro. ${ }^{41}$

Les chemins du billet à l'article (ou au chapitre) sont donc divers : republication telle quelle ou avec une réécriture mineure; rédaction d'un article enrichi à partir billet ; écriture d'un texte sous une première forme plus narrative avant réutilisation des matériaux sous une forme plus analytique. Mes billets sur le déminage pourront ainsi être intégrés dans un mémoire d'habilitation, ou devenir un article à part entière (en revoyant de style pour le «des-oraliser », en modifiant surtout la ponctuation et le rythme des phrases). La perte de l'unité narrative est d'autant plus envisageable que la version d'origine demeure intacte sur le carnet.

Ainsi, aux questions traditionnelles des chercheurs (publier plusieurs articles? dans quelles revues? publier un article ou directement un livre? et dans quelle langue ?) s'ajoute désormais la possibilité de l'écriture de blog. Ces pratiques de tuilage entre publications ne nous sont pas nouvelles. Il faut toutefois ajouter à cette économie la possible circulation entre blog et grands médias.

\section{L'actualité et le lien avec les grands médias}

La réactivité à l'actualité qui caractérise le blog peut conduire à la republication de billets originaux dans la presse, parfois par le biais d'une traduction ou d'une réécriture.

C'est le cas d'un billet écrit immédiatement après l'assassinat du randonneur français Hervé Gourdel dans les montages du Djurdjura le 23 septembre 2014. L'événement ravivait les souvenirs de la Décennie noire des années 1990, durant lesquelles le pays avait combattu une insurrection islamiste. J'avais eu par le passé l'idée d'écrire un ouvrage sur

40 B.T. WHITE, « Quarantine and confinement at Point Nepean », Singular Things, 18 août 2017, [En ligne]. <https://singularthings.wordpress.com/2017/08/18/quarantine-and-confinement-at-point-nepean/>; et « North Head quarantine station », Singular Things, 16 septembre 2017, [En ligne]

<https://singularthings.wordpress.com/2017/09/16/north-head-quarantine-station/> [Consultés le 25 novembre 2019]. Le chapitre paraîtra dans un ouvrage collectif intitule Structures of Protection: Rethinking Refugee Shelter, à paraître en mai 2020.

41 M. RAHAL, « Fille d'Octobre », Textures du temps- حبكات الزمن, 6 juillet 2013,

<http://texturesdutemps.hypotheses.org/826> [Consulté le 23 juillet 2013] ; Republié par la suite : M. RAHAL, « Fille d'Octobre. Générations, engagement et histoire », L'Année du Maghreb, 2014, p. 183-187. 
la forêt où Hervé Gourdel avait été exécuté et face au désarroi, j'ai utilisé les documents et réflexions d'alors pour rédiger un texte, mis en ligne deux jours après l'annonce de l'assassinat ${ }^{42}$. Son devenir est exemplaire de celui de plusieurs autres publiés d'abord sur Textures: suivant les conseils de collègues lecteurs, une version anglaise a été proposée à The Nation, qui l'a publiée ${ }^{43}$. L'idée de cette publication dans le plus ancien hebdomadaire américain n'a pu naître que parce que le carnet en a accueilli une première version suscitant conseils et encouragements. D'autres itérations du même texte sont également parues dans Le Monde et El Watan. ${ }^{44} \mathrm{D}$ 'autres billets de Textures, rédigés par différents auteurs ont été republiés dans Al-Akhbar, L'Humanitéou la revue Bidayat.

Cette circulation des textes du blog vers la presse renforce la posture des chercheurs dans leurs relations aux médias et leur permet de mieux défendre une écriture centrée sur les questions qui les préoccupent, plutôt que sur celles intéressant les journalistes, tout en réagissant aussi à une actualité moins médiatique. Si, pour certains, l'accès à la presse va de soi, l'assurance ou le capital social nécessaires le rendent difficile pour d'autres. Or, la préexistence du texte ouvre des portes que l'on n'oserait sinon pousser.

La circulation des textes se fait parfois dans l'autre sens, du média vers le blog, pour rendre accessible la version longue d'un texte, rendre plus pérenne l'accès à un article de journal ou le donner à lire à un public plus large. C'est l'un des usages que fait Kmar Bendana de HCTC où elle propose ses articles déjà parus dans la presse tunisienne ou algérienne, ou dans des revues papier comme la revue tunisienne Ibla qui n'existe pas en ligne. Le blog s'appare alors à un site personnel conservant la trace d'activités d'abord destinées à d'autres supports.

\section{L'écriture démultipliée}

On pourrait raconter l'histoire de chaque billet de Textures, avec ses inspirations, ses étapes, ses circulations après mise en ligne, les réactions de ses lecteurs et ses résurgences ultérieures. Il y a d'ailleurs moins un lectorat du blog qu'un lectorat spécifique à chaque billet. Ces histoires racontent la démultiplication des versions d'un même texte sur des supports différents.

Cette démultiplication n'est cependant pas réservée au devenir d'un billet. Elle apparaît en fait dès la rédaction, révélant une autre transformation des pratiques d'écriture. On peut en effet retrouver les racines de certains billets dans des posts Facebook, ou des Tweets de leurs auteurs. C'est le cas du billet concernant l'exécution d'Hervé Gourdel, dont plusieurs phrases ont d'abord été des posts Facebook avant que naisse l'idée du billet quelques heures plus tard. Par ailleurs, le compte Twitter de Benjamin T. White (@rain_later) et son blog Singular Things révèlent que la rédaction d'un billet ou d'un fil

\footnotetext{
42 M. RAHAL, « Ici et maintenant - Après l'assassinat d'Hervé Gourdel », Textures du temps- حبكات الزمن 25 septembre 2014, <http://texturesdutemps.hypotheses.org/1241> [Consulté le 20 mars 2015].

43 M. RAHAL, « Into the Woods », The Nation, 31 mars 2015, [En ligne].

<http://www.thenation.com/article/202985/woods>. [Consulté le 24 avril 2015].

44 M. RAHAL, « Où va l'Algérie après l'assassinat d'Hervé Gourdel ? », Le Monde, 1 octobre 2014, [En ligne]. <https://www.lemonde.fr/idees/article/2014/10/01/ou-va-l-algerie-apres-l-assassinat-d-hervegourdel_4498090_3232.html>. [Consulté le 18 décembre 2018] ; M. RAHAL, « Il ne faut pas laisser la sidération nous regagner », El Watan, 10 mars 2014.
} 
de Tweets jouent pour l'auteur des rôles similaires, voire que les deux pratiques peuvent être concurrentes ou substituables selon le temps disponible. Dans son cas, fils Twitter ou billets de blogs peuvent ensuite être devenir des interventions médiatiques ou des publications scientifiques, à l'instar de la lecture critique d'un ouvrage ${ }^{45}$.

\section{CONCLUSION}

Depuis 2012, l'expérience de Textures du Temps révèle l'écriture de blog comme une pratique multiforme et expérimentale, dont il est difficile de tirer des lois du genre. S'il est vrai que le carnet de recherche donne à voir l'histoire en train de se faire (et peut alors modifier le cours d'un travail de recherche), la question de la temporalité de cette écriture en ligne est plus complexe. Certes, beaucoup de billets permettent un passage à la publication à un stade plus précoce que la rédaction d'un article de revue ou d'un livre. Mais l'écriture libérée de certains aspects formels, l'individualité de la démarche, la possibilité d'une écrire à la première personne en font aussi l'occasion d'un pas de côté réflexif qui peut se dérouler avant, pendant ou même après la rédaction plus classique.

L'écriture de carnet de recherche s'inscrit désormais dans une constellation d'écritures, avec des formes et sur les supports démultipliés, des bribes de textes - porteurs de sources, archives ou idées - circulant des uns aux autres avec des influences mutuelles. Ces circulations nouvelles doivent être pensées en même temps que toutes les questions éthiques et politiques attachées à la circulation et à l'accessibilité du savoir, y compris celle du risque que présente la dépendance à l'égard des GAFA, la politique marchande des grands éditeurs de revues scientifiques ou les circulations Nord-Sud et Sud-Nord des résultats de la recherche.

Au sortir de cette exploration, des questions demeurent. L'une d'elle concerne le nombre d'historiens actuellement impliqués dans ces pratiques nouvelles d'écritures, en France et dans le monde. La seconde concerne les pratiques de lecture des blogs d'histoire par les historiens eux-mêmes ainsi que les pratiques de citations des billets. L'une et l'autre nous permettraient d'avoir une idée plus juste de l'ampleur des transformations en cours dans le métier des historiens.

45 Le fil Twitter : <https://twitter.com/rain later/status/852153866774880256?s=19>

[Consulté le 22 novembre 2019]. Il est devenu un compte-rendu dans une revue : B.T. WHITE, « Refuge and History: A Critical Reading of a Polemic », Migration and Society, juin 2019, p. 107-118. 